\title{
COUPLING FINITE ELEMENT AND SPECTRAL METHODS: FIRST RESULTS
}

\author{
CHRISTINE BERNARDI, NAÏMA DEBIT, AND YVON MADAY
}

\begin{abstract}
A Poisson equation on a rectangular domain is solved by coupling two methods: the domain is divided in two squares; a finite element approximation is used on the first square and a spectral discretization is used on the second. Two kinds of matching conditions on the interface are presented and compared; in both cases, error estimates are proved.
\end{abstract}

\section{INTRODUCTION}

To approximate the solutions of partial differential equations, a number of methods can be successfully applied: among them, spectral-type methods, in which the discrete solution is a polynomial of high degree, are known to be very accurate when the solution to be approximated is very smooth (see $[16,8]$ for a general description of these methods). Their main drawback lies in the difficulty to take into account the singularities of the function to be approximated, and also in the difficulty in handling domains with a complicated boundary. This last problem is usually solved by decomposition into subdomains and/or transformation of coordinates. On the other hand, the finite element method, where the discrete solution, restricted to very small domains called "elements", is a polynomial of low degree, is well suited to problems with complex geometries, but its accuracy is limited by the degree of the polynomials (general properties of finite elements are analyzed in [11]). Several attempts have been made to combine the two methods into a unified framework, and thereby obtaining the advantages of both. The main idea on which these attempts rely consists of a decomposition of the domain into (rather) small subdomains so as to fit the geometric complexity of the boundary, and then use high-degree polynomials on each subdomain to approximate the solution. Two different approaches have been proposed: the spectral element method and the $p$-version of the finite element method. The spectral element method [25], which consists of using a spectral algorithm on a fixed number of subdomains, is presently developed for a growing number of problems (see, for instance, [15, 19, and 23]); on the opposite side, the so-called $p$-version of the finite element method, where

Received September 28, 1987; revised January 16, 1989.

1980 Mathematics Subject Classification (1985 Revision). Primary 65N30, 65N35; Secondary $35 \mathrm{~J} 05,65 \mathrm{~N} 15$.

Research was supported in part for the last author by the National Aeronautics and Space Administration under NASA Contract No NAS1-18107 while he was in residence at ICASE, NASA Langley Research Center, Hampton, VA 23665. 


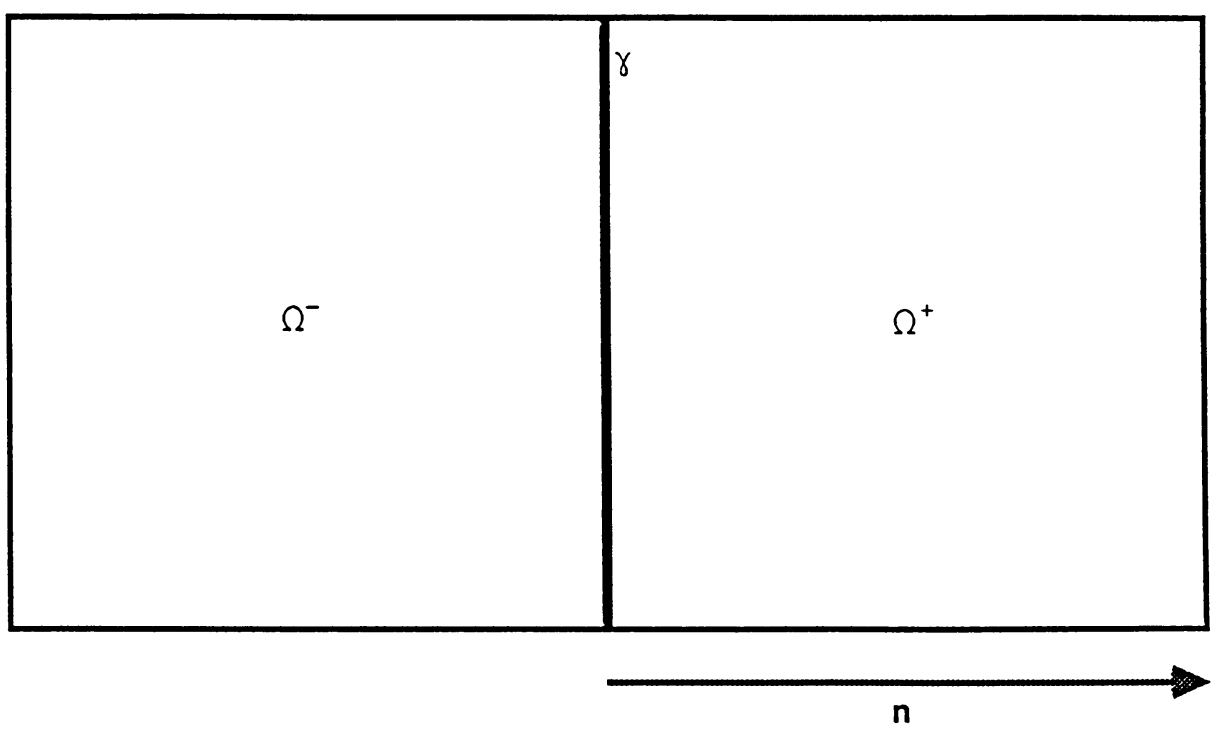

FIGURE 1.1. Decomposition of the domain.

the analysis of the consistency error and of the approximation error. The final error estimates, together with concluding remarks, are given in $\S 5$.

The main results of this paper were first presented in [5].

Notation. Let $\Delta$ denote any open interval of the real line or any domain in $\mathbb{R}^{2}$ with a polygonal boundary. For any real number $s$, we consider the classical Hilbert Sobolev spaces $H^{s}(\Delta)$, provided with the usual norm $\|\cdot\|_{s, \Delta}$, and also, when $s$ is an integer, with the seminorm $|\cdot|_{s, \Delta}$. For any real number $s \geq 0$ and any $p, 1 \leq p \leq+\infty, p \neq 2$, we also use the Sobolev spaces $W^{s, p}(\Delta)$, which are no longer Hilbert spaces, provided with the norm $\|\cdot\|_{s, p, \Delta}$. Finally, for any real number $s \geq 0, H_{0}^{s}(\Delta)$ stands for the closure in $H^{s}(\Delta)$ of the space of infinitely differentiable functions with compact support in $\Delta$.

Throughout this paper, with any function $v$ defined on $\Omega$, we associate the pair $v^{*}=\left(v^{-}, v^{+}\right)$, where $v^{-}$(resp. $\left.v^{+}\right)$denotes the restriction of $v$ to $\Omega^{-}$ (resp. $\left.\Omega^{+}\right)$. The scalar product on $L^{2}\left(\Omega^{-}\right) \times L^{2}\left(\Omega^{+}\right)$,

$$
\left(u^{*}, v^{*}\right)=\int_{\Omega^{-}} u^{-}(\mathbf{x}) v^{-}(\mathbf{x}) d \mathbf{x}+\int_{\Omega^{+}} u^{+}(\mathbf{x}) v^{+}(\mathbf{x}) d \mathbf{x},
$$

coincides with the usual one on $L^{2}(\Omega)$. We also provide the product $H^{1}\left(\Omega^{-}\right) \times$ $H^{1}\left(\Omega^{+}\right)$with the norm

$$
\left\|v^{*}\right\|=\left[\left(v^{*}, v^{*}\right)+\left(\nabla v^{*}, \nabla v^{*}\right)\right]^{1 / 2}
$$

the space of pairs $v^{*}$ in $H^{1}\left(\Omega^{-}\right) \times H^{1}\left(\Omega^{+}\right)$with $v$ continuous through $\gamma$ is isomorphic to $H^{1}(\Omega)$. Finally, we define on $H^{1}\left(\Omega^{-}\right) \times H^{1}\left(\Omega^{+}\right)$the bilinear form

$$
a\left(u^{*}, v^{*}\right)=\left(\nabla u^{*}, \nabla v^{*}\right) \quad \forall\left(u^{*}, v^{*}\right) \in\left[H^{1}\left(\Omega^{-}\right) \times H^{1}\left(\Omega^{+}\right)\right]^{2} .
$$


Clearly, for any function $f$ in $L^{2}(\Omega)$, problem $(1.1)$ is equivalent to the following one: Find $u$ in $H_{0}^{1}(\Omega)$ such that

$$
a\left(u^{*}, v^{*}\right)=\left(f^{*}, v^{*}\right) \quad \forall v \in H_{0}^{1}(\Omega) .
$$

This variational form is precisely the one which will be used in order to define the discrete problems.

In all that follows, $c, c^{\prime}, c^{\prime \prime}, \ldots$ are generic positive constants independent of the discretization parameters.

\section{THE DISCRETE SPACES AND PROBLEMS}

2.1. Definition of the discrete spaces. We have to define a discrete space on each subdomain $\Omega^{-}$and $\Omega^{+}$, and then we must make precise the matching conditions on the interface.

Let $h$ be a real parameter, $0<h \leq 1$, which will tend to 0 . With each value of $h$, we associate a triangulation $\mathscr{T}_{h}$ of the domain $\Omega^{-}$, i.e., a finite set of triangles such that the intersection of two triangles is either empty, or a vertex, or an edge, and such that (cf. Figure 2.1)

$$
\bar{\Omega}^{-}=\bigcup_{K \in \mathscr{T}_{h}} K ;
$$

$h$ is the upper bound of the diameters of the triangles of $\mathscr{T}_{h}$. We denote by $h_{K}$ the diameter of any triangle $K$ in $\mathscr{T}_{h}$, and by $\rho_{K}$ the diameter of the inscribed circle in $K$. Next, we assume that the family $\left(\mathscr{T}_{h}\right)_{h}$ is regular in the following sense (cf. [11, §3.1] or [3, Definition 3.1]): there exists a constant $\tau>0$ such that, for any $h$ and for any $K$ in $\mathscr{T}_{h}$, the following inequality holds:

$$
\rho_{K} \geq \tau h_{K} \text {. }
$$

Let $k$ be a fixed integer $\geq 1$. For any closed subset $A$ of $\mathbb{R}\left(\right.$ resp. $\mathbb{R}^{2}$ ), we denote by $P_{k}(A)$ the set of the restrictions to $A$ of polynomials of one variable (resp. two variables) with total degree $\leq k$. With any triangulation $\mathscr{T}_{h}$, we have the associated finite-dimensional space $X_{h}$ defined by

$$
X_{h}=\left\{v_{h} \in \mathscr{C}^{0}\left(\bar{\Omega}^{-}\right) ; \forall K \in \mathscr{T}_{h}, v_{h \mid K} \in P_{k}(K) \text { and } v_{h}=0 \text { on } \partial \Omega^{-} \backslash \gamma\right\} \text {. }
$$

We also need the finite-dimensional trace space

$$
x_{h}=\left\{v_{h \mid \gamma}, v_{h} \in X_{h}\right\} \text {. }
$$

In order to build an appropriate basis of $X_{h}$ and $x_{h}$, we consider each triangle $K$ as the support of a Lagrange finite element $\left(K, P_{k}(K), \Xi_{K}\right)$, where $\Xi_{K}$ is the set of all points in $K$ with barycentric coordinates $i / k, j / k$, and $(k-i-j) / k$, $0 \leq i, j \leq k, i+j \leq k$; it is well known [11, Theorem 2.2.1] that this set of points is $P_{k}(K)$-unisolvent. Next, we set

$$
\Xi_{h}=\bigcup_{K \in J_{h}} \Xi_{K},
$$

and also

$$
\xi_{h}=\left\{\mathbf{a} \in \Xi_{h} \cap \gamma\right\}
$$


With each point a in $\Xi_{h} \cap\left(\Omega^{-} \cup \gamma\right)$, we associate the unique function $q_{\mathrm{a}}$ of $X_{h}$ which is equal to 1 in a and vanishes at any other point of $\Xi_{h}$. Then the set $\left\{q_{\mathbf{a}}, \mathbf{a} \in \Xi_{h} \cap\left(\Omega^{-} \cup \gamma\right)\right\}$ is a basis of the space $X_{h}$ and the set $\left\{q_{\mathbf{a} \mid \gamma}, \mathbf{a} \in \xi_{h}\right\}$ is a basis of the space $x_{h}$.

Next, let $N$ be an integer $\geq 1$, which will tend to $+\infty$. For any integer $n \geq 0$, we denote by $Q_{n}\left(\bar{\Omega}^{+}\right)$the set of the restrictions to $\bar{\Omega}^{+}$of polynomials of two variables with degree $\leq n$ with respect to each variable. For each integer $N$, we consider the finite-dimensional space $X_{N}$ defined by

$$
X_{N}=\left\{v_{N} \in Q_{N}\left(\bar{\Omega}^{+}\right) ; v_{N}=0 \text { on } \partial \Omega^{+} \backslash \gamma\right\} .
$$

Let $\left(L_{n}\right)_{n \in \mathbb{N}}$ be the family of Legendre polynomials on $[0,1]$, i.e., of orthogonal polynomials on $[0,1]$ such that $L_{n}, n \in \mathbb{N}$, is of degree $n$ and satisfies $L_{n}(0)=1$. We recall that the set $\left\{L_{m} \otimes L_{n}, 0 \leq m, n \leq N\right\}$ is a basis of $Q_{N}\left(\bar{\Omega}^{+}\right)$. However, we shall also characterize the polynomials of $X_{N}$ by pointwise values: let $\zeta_{j}, 0 \leq j \leq N$, be the roots of the polynomial $\zeta(1-\zeta) L_{N}^{\prime}(\zeta)$, with $0=\zeta_{0}<\zeta_{1}<\cdots<\zeta_{N}=1$; we set (cf. Figure 2.1)

$$
\Xi_{N}=\left\{\left(\zeta_{i}, \zeta_{j}\right), 0 \leq i, j \leq N\right\} \text {, }
$$

and

$$
\xi_{N}=\Xi_{N} \cap \gamma=\left\{\left(0, \zeta_{j}\right), 1 \leq j \leq N-1\right\}
$$

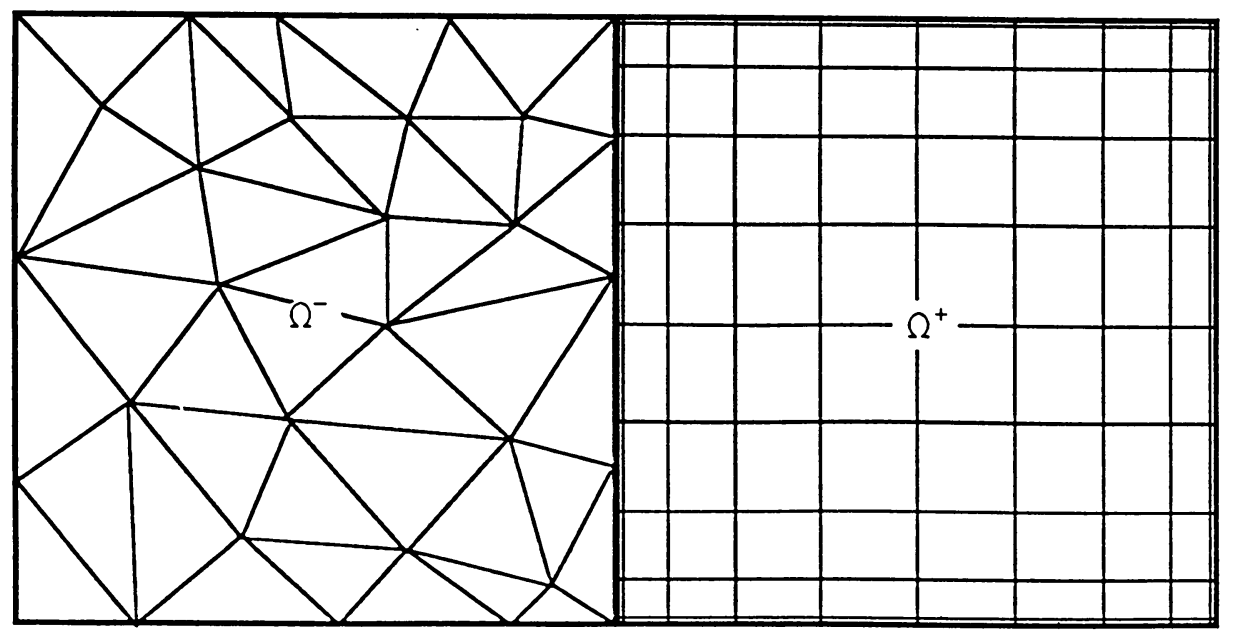

Figure 2.1. The triangulation $\mathscr{T}_{h}$ and the set $\Xi_{N}$.

Finally, with each value of $h$ and $N$, we associate the discretization parameter $\delta=\left(h, N^{-1}\right)$. The pair $u^{*}$, where $u$ is the solution of problem (1.1), will be approximated in a subspace of $X_{h} \times X_{N}$ consisting of all pairs which satisfy a matching condition on the interface $\gamma$. More precisely, we are going to consider two kinds of matching conditions, with which we associate two kinds 
of discrete spaces, both denoted by $V_{\delta}$ :

(1) pointwise matching condition: the space $V_{\delta}^{P}$ is defined by

$$
V_{\delta}^{P}=\left\{v_{\delta}^{*}=\left(v_{h}, v_{N}\right) \in X_{h} \times X_{N} ; \forall \mathbf{a} \in \xi_{h}, v_{h}(\mathbf{a})=v_{N}(\mathbf{a})\right\} ;
$$

(2) integral matching condition: the space $V_{\delta}^{I}$ is defined by

$$
\begin{aligned}
V_{\delta}^{I}=\left\{v_{\delta}^{*}=\left(v_{h}, v_{N}\right) \in\right. & X_{h} \times X_{N} ; \forall q_{h} \in x_{h}, \\
& \left.\int_{\gamma}\left(v_{h}-v_{N}\right)(0, y) q_{h}(y) d y=0\right\} .
\end{aligned}
$$

Here, we compare the two kinds of spaces; the integral matching condition will turn out to be better.

Remark 2.1. We immediately note that both methods are nonconforming since a function $v_{\delta}$, associated with a pair $v_{\delta}^{*}$ of $V_{\delta}$, is generally discontinuous through $\gamma$ and consequently does not belong to $H_{0}^{1}(\Omega)$. Indeed, for $N \geq k$, the function $v_{\delta}$ associated with the pair $v_{\delta}^{*}=\left(v_{h}, v_{N}\right)$ in $X_{h} \times X_{N}$ belongs to $H_{0}^{1}(\Omega)$ if and only if its restriction to $\gamma$ is a polynomial of $P_{k}(\bar{\gamma}) \cap H_{0}^{1}(\gamma)$; in the particular case $k=1$, this implies that both $v_{h}$ and $v_{N}$ vanish on $\gamma$.

Remark 2.2. From a numerical point of view, to enforce the pointwise matching condition, one has to interpolate polynomials of $X_{N}$ at every point of $\xi_{h}$ and hence must store the values $L_{n}(\mathbf{a}), 0 \leq n \leq N, \mathbf{a} \in \xi_{h}$. On the other hand, to enforce the integral matching condition, one needs to store the integrals $\int_{y} L_{n}(y) q_{\mathbf{a}}(0, y) d y, 0 \leq n \leq N, \mathbf{a} \in \xi_{h}$. Consequently, the costs of the two methods are of the same order.

However, when $k$ is equal to 1 , for a given value of $N$, it is possible to choose the triangulation $\mathscr{T}_{h}$ such that the sets $\xi_{h}$ and $\xi_{N}$ coincide. Then, since the polynomials of $X_{N}$ are characterized by their values at the points of $\Xi_{N}$, enforcing the pointwise matching condition would be less expensive. But this would require very strong restrictions on the triangulation $\mathscr{T}_{h}$; in particular, the parameters $h$ and $N$ would be linked by a relation of the type $h \geq c N^{-1}$. Moreover, the triangulation could not be uniformly regular since it is well known (see [30, Theorem 6.21.3]) that the $\zeta_{j}, 1 \leq j \leq N-1$, satisfy $\zeta_{j}=\sin ^{2} \theta_{j}$ with $(2 j-1) \pi / 4 N<\theta_{j}<(j+1) \pi / 2(N+1)$; hence, the points of $\xi_{N}$ are not at all equally distributed (they cluster near +1 ). That is why we do not recommend such a choice.

2.2. The discrete problems. We are now in a position to define the discrete problems. We recall (see $[13, \S 2.7$ or 18 , Chapter 25$]$ ) that there exist positive weights $\rho_{j}, 0 \leq j \leq N$, such that the Gauss-Lobatto quadrature formula

$$
\int_{0}^{1} \Phi(\zeta) d \zeta \simeq \sum_{j=0}^{N} \Phi\left(\zeta_{j}\right) \rho_{j}
$$

is exact for all polynomials of degree $\leq 2 N-1$. 
With each point $\mathbf{a}=\left(\zeta_{i}, \zeta_{j}\right)$ in $\Xi_{N}$, we associate the weight $\rho_{\mathbf{a}}=\rho_{i} \rho_{j}$. We now introduce the following discrete bilinear form on $L^{2}\left(\Omega^{-}\right) \times \mathscr{C}^{0}\left(\bar{\Omega}^{+}\right)$:

$$
\left(u^{*}, v^{*}\right)_{\delta}=\sum_{K \in \mathscr{F}_{h}} \int_{K} u^{-}(\mathbf{x}) v^{-}(\mathbf{x}) d \mathbf{x}+\sum_{\mathbf{a} \in \Xi_{N}} u^{+}(\mathbf{a}) v^{+}(\mathbf{a}) \rho_{\mathbf{a}},
$$

which coincides with the usual scalar product on $L^{2}\left(\Omega^{-}\right) \times Q_{N-1}\left(\bar{\Omega}^{+}\right)$. Finally, we define on $H^{1}\left(\Omega^{-}\right) \times \mathscr{C}^{1}\left(\bar{\Omega}^{+}\right)$the bilinear form

$$
a_{\delta}\left(u^{*}, v^{*}\right)=\left(\nabla u^{*}, \nabla v^{*}\right)_{\delta} \quad \forall\left(u^{*}, v^{*}\right) \in\left[H^{1}\left(\Omega^{-}\right) \times \mathscr{C}^{1}\left(\bar{\Omega}^{+}\right)\right]^{2} .
$$

Then, for any pair $f^{*}$ given in $L^{2}\left(\Omega^{-}\right) \times \mathscr{C}^{0}\left(\bar{\Omega}^{+}\right)$, for each kind of matching condition, the discrete problem is the following: Find $u_{\delta}$, with $u_{\delta}^{*}$ in $V_{\delta}$, such that

$$
a_{\delta}\left(u_{\delta}^{*}, v_{\delta}^{*}\right)=\left(f^{*}, v_{\delta}^{*}\right)_{\delta} \quad \forall v_{\delta}^{*} \in V_{\delta} .
$$

Remark 2.3. Of course, in definition (2.13), one could, by using a quadrature formula, replace each integral $\int_{K} u^{-}(\mathbf{x}) v^{-}(\mathbf{x}) d \mathbf{x}$ by its approximation. The resulting algorithm will be thoroughly analyzed in [14].

We recall $[9$, Lemma 3.2] the property

$$
\sum_{j=0}^{N} L_{N}\left(\zeta_{j}\right)^{2} \rho_{j}=\left(2+N^{-1}\right) \int_{0}^{1} L_{N}(\zeta)^{2} d \zeta
$$

Since the quadrature formula $(2.12)$ is exact for all polynomials of degree $\leq$ $2 N-1$, the discrete scalar product $(\cdot, \cdot)_{\delta}$ is uniformly equivalent to $(\cdot, \cdot)$ on $L^{2}\left(\Omega^{-}\right) \times Q_{N}\left(\bar{\Omega}^{+}\right)$. Consequently, the form $a_{\delta}$ satisfies the following properties of continuity:

$$
\left|a_{\delta}\left(u^{*}, v^{*}\right)\right| \leq c\left\|u^{*}\right\|\left\|v^{*}\right\| \quad \forall\left(u^{*}, v^{*}\right) \in\left[H^{1}\left(\Omega^{-}\right) \times Q_{N}\left(\bar{\Omega}^{+}\right)\right]^{2},
$$

and of ellipticity:

$$
a_{\delta}\left(u^{*}, u^{*}\right) \geq\left(\nabla u^{*}, \nabla u^{*}\right) \quad \forall u^{*} \in H^{1}\left(\Omega^{-}\right) \times Q_{N}\left(\bar{\Omega}^{+}\right) .
$$

Now, since both $\partial \Omega^{-} \cap \partial \Omega$ and $\partial \Omega^{+} \cap \partial \Omega$ have a positive measure, it follows from the Poincaré-Friedrichs inequality that the seminorm $v^{*} \rightarrow\left(\nabla v^{*}, \nabla v^{*}\right)^{1 / 2}$ is a norm equivalent to $\|\cdot\|$ on the space

$$
\left\{v^{*} \in H^{1}\left(\Omega^{-}\right) \times H^{1}\left(\Omega^{+}\right) ; v=0 \text { on } \partial \Omega\right\},
$$

which yields in our particular case

$$
a_{\delta}\left(u^{*}, u^{*}\right) \geq c\left\|u^{*}\right\|^{2} \quad \forall u^{*} \in H^{1}\left(\Omega^{-}\right) \times Q_{N}\left(\bar{\Omega}^{+}\right) .
$$

Thus we have proved the following result.

Proposition 2.4. For pointwise as well as integral matching conditions, problem (2.15) has a unique solution $u_{\delta}$ with $u_{\delta}^{*}$ in $V_{\delta}$.

The purpose of what follows is to give an estimate for the error between the solutions $u$ and $u_{\delta}$ of problems (1.1) and (2.15), respectively. We begin with a classical bound (see [11, Theorem 4.2.2]). 
Proposition 2.5. For pointwise as well as integral matching conditions, the solutions $u$ and $u_{\delta}$ of problems (1.1) and (2.15) satisfy

$$
\begin{aligned}
\left\|u^{*}-u_{\delta}^{*}\right\| \leq c & \left\{\inf _{v_{\delta}^{*} \in V_{\delta}}\left\{\left\|u^{*}-v_{\delta}^{*}\right\|+\sup _{w_{\delta}^{*} \in V_{\delta}}\left[a\left(v_{\delta}^{*}, w_{\delta}^{*}\right)-a_{\delta}\left(v_{\delta}^{*}, w_{\delta}^{*}\right)\right] /\left\|w_{\delta}^{*}\right\|\right\}\right. \\
& +\sup _{w_{\delta}^{*} \in V_{\delta}}\left[\left(f^{*}, w_{\delta}^{*}\right)-\left(f^{*}, w_{\delta}^{*}\right)_{\delta}\right] /\left\|w_{\delta}^{*}\right\| \\
& \left.+\sup _{w_{\delta}^{*}=\left(w_{h}, w_{N}\right) \in V_{\delta}} \int_{\gamma}(\partial u / \partial n)(0, y)\left(w_{N}-w_{h}\right)(0, y) d y /\left\|w_{\delta}^{*}\right\|\right\} .
\end{aligned}
$$

Proof. Let $v_{\delta}^{*}$ be any element in $V_{\delta}$. Using (2.19), we have

$$
\begin{aligned}
c\left\|u_{\delta}^{*}-v_{\delta}^{*}\right\|^{2} \leq & a_{\delta}\left(u_{\delta}^{*}-v_{\delta}^{*}, u_{\delta}^{*}-v_{\delta}^{*}\right) \\
=-a\left(v_{\delta}^{*}, u_{\delta}^{*}-v_{\delta}^{*}\right)+a\left(v_{\delta}^{*}, u_{\delta}^{*}-v_{\delta}^{*}\right) & \quad-a_{\delta}\left(v_{\delta}^{*}, u_{\delta}^{*}-v_{\delta}^{*}\right)+\left(f^{*}, u_{\delta}^{*}-v_{\delta}^{*}\right)_{\delta} .
\end{aligned}
$$

Next, it follows from (1.1) that, for any $w^{*}$ in $H^{1}\left(\Omega^{-}\right) \times H^{1}\left(\Omega^{+}\right)$such that $w$ vanishes on $\partial \Omega$,

$$
\begin{aligned}
\left(f^{*}, w^{*}\right) & =\int_{\Omega} f(\mathbf{x}) w(\mathbf{x}) d \mathbf{x}=-\int_{\Omega^{-}}(\Delta u)(\mathbf{x}) w^{-}(\mathbf{x}) d \mathbf{x}-\int_{\Omega^{+}}(\Delta u)(\mathbf{x}) w^{+}(\mathbf{x}) d \mathbf{x} \\
& =a\left(u^{*}, w^{*}\right)+\int_{\gamma}(\partial u / \partial n)(0, y)\left(w^{+}-w^{-}\right)(0, y) d y .
\end{aligned}
$$

Setting $w^{*}=u_{\delta}^{*}-v_{\delta}^{*}=\left(w_{h}, w_{N}\right)$ and combining this result with the previous inequality, we obtain

$$
\begin{aligned}
c\left\|u_{\delta}^{*}-v_{\delta}^{*}\right\|^{2} \leq & a\left(u^{*}-v_{\delta}^{*}, u_{\delta}^{*}-v_{\delta}^{*}\right)+a_{\delta}\left(v_{\delta}^{*}, u_{\delta}^{*}-v_{\delta}^{*}\right)-a_{\delta}\left(v_{\delta}^{*}, u_{\delta}^{*}-v_{\delta}^{*}\right) \\
& -\left(f^{*}, u_{\delta}^{*}-v_{\delta}^{*}\right)+\left(f^{*}, u_{\delta}^{*}-v_{\delta}^{*}\right)_{\delta} \\
& +\int_{\gamma}(\partial u / \partial n)(0, y)\left(w_{N}-w_{h}\right)(0, y) d y,
\end{aligned}
$$

and (2.20) follows.

We are now interested in deriving a bound for

(1) the consistency error term

$$
\sup _{w_{\delta}^{*}=\left(w_{h}, w_{N}\right) \in V_{\delta}} \int_{\gamma}(\partial u / \partial n)(0, y)\left(w_{N}-w_{h}\right)(0, y) d y /\left\|w_{\delta}^{*}\right\|,
$$

(2) the approximation error term

$$
\inf _{v_{\delta}^{*} \in V_{\delta}}\left\|u^{*}-v_{\delta}^{*}\right\|
$$

since estimating the other terms in (2.20) is standard in spectral methods.

\section{ANALYSIS OF THE CONSISTENCY ERROR}

The aim of this section is to study the term

$$
\int_{y}(\partial u / \partial n)(0, y)\left(w_{N}-w_{h}\right)(0, y) d y
$$


for any pair $w_{\delta}^{*}=\left(w_{h}, w_{N}\right)$ in $V_{\delta}$, where $u$ is a given function on $\Omega$ which we shall assume to be sufficiently smooth. The analysis involves only onedimensional approximation operators.

3.1. Pointwise matching. We recall that there exists an interpolation operator $i_{h}$ from $\left\{v \in \mathscr{C}^{0}(\bar{\gamma}) ; v(0)=v(1)=0\right\}$ into $x_{h}$ such that, for any function $v$ continuous on $\bar{\gamma}$ and vanishing at 0 and $1, i_{h} v$ is the only element of $x_{h}$ which satisfies

$$
\left(i_{h} v\right)(\mathbf{a})=v(\mathbf{a}) \quad \forall \mathbf{a} \in \xi_{h} .
$$

Moreover, there exists a constant $c$ such that, for any real number $\varepsilon, 0<\varepsilon \leq$ $1 / 2$, if the function $v$ belongs to $H^{1 / 2+\varepsilon}(\gamma)$, the following interpolation error estimate holds [4]:

$$
\left\|v-i_{h} v\right\|_{0, \gamma} \leq(c / \sqrt{\varepsilon}) h^{1 / 2+\varepsilon}\|v\|_{1 / 2+\varepsilon, \gamma} .
$$

We are now in a position to prove the following result.

Proposition 3.1. For any function $u$ in $H_{0}^{1}(\Omega) \cap H^{2}(\Omega)$, the following estimate holds for any $w_{\delta}^{*}=\left(w_{h}, w_{N}\right)$ in $V_{\delta}^{P}$ :

$$
\int_{\gamma}(\partial u / \partial n)(0, y)\left(w_{N}-w_{h}\right)(0, y) d y \leq c h^{1 / 2} \sqrt{\left|\log \left(h N^{2}\right)\right|}\left\|u^{-}\right\|_{2, \Omega^{-}}\left\|w_{\delta}^{*}\right\| .
$$

Proof. Since $u$ belongs to $H^{2}(\Omega)$, the trace $\partial u / \partial n$ belongs to $H^{1 / 2}(\gamma)$, hence we have

$$
\|\partial u / \partial n\|_{1 / 2, \gamma} \leq c\|u\|_{2, \Omega^{-}}
$$

Next, we estimate

$$
\begin{aligned}
\int_{\gamma}(\partial u / \partial n)(0, y)\left(w_{N}-w_{h}\right)(0, y) d y & \leq\|\partial u / \partial n\|_{0, \gamma}\left\|w_{h}-w_{N}\right\|_{0, \gamma} \\
& \leq c\|u\|_{2, \Omega^{-}}\left\|w_{h}-w_{N}\right\|_{0, \gamma} .
\end{aligned}
$$

But, in view of the definition (2.10) of $V_{\delta}^{P}, w_{h}$ is equal to $i_{h} w_{N}$ on $\gamma$, so that by (3.2),

$$
\int_{\gamma}(\partial u / \partial n)(0, y)\left(w_{N}-w_{h}\right)(0, y) d y \leq\left(c^{\prime} / \sqrt{\varepsilon}\right) h^{1 / 2+\varepsilon}\|u\|_{2, \Omega^{-}}\left\|w_{N}\right\|_{1 / 2+\varepsilon, \gamma} .
$$

Applying the inverse inequality [9] gives

$$
\int_{\gamma}(\partial u / \partial n)(0, y)\left(w_{N}-w_{h}\right)(0, y) d y \leq\left(c^{\prime} / \sqrt{\varepsilon}\right) N^{2 \varepsilon} h^{1 / 2+\varepsilon}\|u\|_{2, \Omega^{-}}\left\|w_{N}\right\|_{1 / 2, \gamma} .
$$

Choosing $\varepsilon=1 /\left|\log \left(h N^{2}\right)\right|$, we obtain the desired result.

Remark 3.2. Of course, the estimate (3.3) is not what we want, since convergence is obtained only if the discretization parameters are linked by the following condition:

$$
\lim \left[h^{1 / 2} \sqrt{\left|\log \left(h N^{2}\right)\right|}\right]=0
$$


(in fact, in (3.3) and in this condition, $h$ can be replaced by $\tilde{h}$ which is the greatest of the lengths of the edges of triangles $K$ in $\mathscr{T}_{h}$ contained in $\bar{\gamma}$ ).

Remark 3.3. The estimate (3.3) is independent of $k$; indeed, we do not know how to improve it for large values of $k$.

3.2. Integral matching. This case turns out to be simpler. We denote by $\pi_{h}$ the orthogonal projection operator from $L^{2}(\gamma)$ onto $x_{h}$. We have for any $v$ in $H_{0}^{1}(\gamma)$,

$$
\left\|v-\pi_{h} v\right\|_{0, \gamma} \leq\left\|v-i_{h} v\right\|_{0, \gamma},
$$

so that, for any $v$ in $H_{0}^{1}(\gamma) \cap H^{l}(\gamma), 1 \leq l \leq k+1$,

$$
\left\|v-\pi_{h} v\right\|_{0, \gamma} \leq c h^{l}\|v\|_{l, \gamma} \text {. }
$$

By interpolation, this inequality also holds for any $v$ in $H_{0}^{l}(\gamma), 1 / 2<l<1$. Finally, recalling that the interpolation space with index $1 / 2$ between $H_{0}^{1}(\gamma)$ and $L^{2}(\gamma)$ is $H_{00}^{1 / 2}(\gamma)$ (see [20, Chapter 1, Theorem 11.7]) and denoting by $\|\cdot\|_{1 / 2^{*}, \gamma}$ the norm of $H_{00}^{1 / 2}(\gamma)$, we also obtain for any $v$ in $H_{00}^{1 / 2}(\gamma)$,

$$
\left\|v-\pi_{h} v\right\|_{0, \gamma} \leq \operatorname{ch}^{1 / 2}\|v\|_{1 / 2^{*}, \gamma} .
$$

Now, we prove the following.

Proposition 3.4. For any function $u$ in $H_{0}^{1}(\Omega) \cap H^{2}(\Omega)$ such that the function $u^{-}$belongs to $H^{l}\left(\Omega^{-}\right)$, where $l$ is a real number, $2 \leq l \leq k+5 / 2$, the following estimate holds for any $w_{\delta}^{*}=\left(w_{h}, w_{N}\right)$ in $V_{\delta}^{I}$ :

$$
\int_{\gamma}(\partial u / \partial n)(0, y)\left(w_{N}-w_{h}\right)(0, y) d y \leq c h^{l-1}\left\|u^{-}\right\|_{l, \Omega^{-}}\left\|w_{\delta}^{*}\right\|
$$

Proof. Let $w_{\delta}^{*}=\left(w_{h}, w_{N}\right)$ be any element in $V_{\delta}^{I}$; by the definition (2.11) of $V_{\delta}^{I}, w_{h}$ coincides with $\pi_{h} w_{N}$ on $\gamma$. We compute

$$
\begin{aligned}
\int_{\gamma}(\partial & u / \partial n)(0, y)\left(w_{N}-w_{h}\right)(0, y) d y \\
& =\int_{\gamma}(\partial u / \partial n)(0, y)\left(w_{N}-\pi_{h} w_{N}\right)(0, y) d y \\
& =\int_{\gamma}\left[(\partial u / \partial n)-\pi_{h}(\partial u / \partial n)\right](0, y)\left(w_{N}-\pi_{h} w_{N}\right)(0, y) d y
\end{aligned}
$$

so that

$$
\begin{aligned}
& \int_{\gamma}(\partial u / \partial n)(0, y)\left(w_{N}-w_{h}\right)(0, y) d y \\
& \quad \leq\left\|(\partial u / \partial n)-\pi_{h}(\partial u / \partial n)\right\|_{0, \gamma}\left\|w_{N}-\pi_{h} w_{N}\right\|_{0, \gamma}
\end{aligned}
$$

We note that $\partial u / \partial n$ belongs to $H_{00}^{1 / 2}(\gamma) \cap H^{l-3 / 2}(\gamma)$ and that, since $w_{N}$ vanishes on $\partial \Omega^{+} \backslash \gamma, w_{N \mid \gamma}$ belongs to $H_{00}^{1 / 2}(\gamma)$. Applying (3.5) or (3.6) to bound 
the first term, and (3.6) to bound the second, we obtain

$$
\int_{\gamma}(\partial u / \partial n)(0, y)\left(w_{N}-w_{h}\right)(0, y) d y \leq c h^{l-1}\|\partial u / \partial n\|_{l-3 / 2, \gamma}\left\|w_{N}\right\|_{1 / 2^{*}, \gamma}
$$

(with $\|\partial u / \partial n\|_{1 / 2, \gamma}$ replaced by $\|\partial u / \partial n\|_{1 / 2^{*}, \gamma}$ in the case $l=2$ ), and the result follows.

Remark 3.5. Clearly, the estimate (3.7) is much better than (3.3), since it is independent of $N$. In fact, the term $\int_{\gamma}(\partial u / \partial n)(0, y)\left(w_{N}-w_{h}\right)(0, y) d y$ goes to 0 whenever the discretization parameter $h$ decreases to 0 .

\section{ANALYSIS OF THE APPROXIMATION ERROR}

We begin by recalling some properties of the approximation by finite element functions and by polynomials in the two-dimensional case.

First, since for each $K$ in $\mathscr{T}_{h}$ the set $\Xi_{K}$ is $P_{k}(K)$-unisolvent, there exists an interpolation operator $\mathscr{I}_{h}$ from $\left\{v \in \mathscr{C}^{0}\left(\bar{\Omega}^{-}\right) ; v=0\right.$ on $\left.\partial \Omega^{-} \backslash \gamma\right\}$ into $X_{h}$ such that, for any function $v$ continuous on $\bar{\Omega}^{-}$and vanishing on $\partial \Omega^{-} \backslash \gamma$, $\mathscr{I}_{h} v$ is the only element of $X_{h}$ which satisfies

$$
\left(\mathscr{I}_{h} v\right)(\mathbf{a})=v(\mathbf{a}) \quad \forall \mathbf{a} \in \Xi_{h} \cap\left(\Omega^{-} \cup \gamma\right) .
$$

Moreover, if the function $v$ belongs to $H^{l}\left(\Omega^{-}\right)$for a real number $l, 2 \leq l \leq$ $k+1$, the following interpolation error estimate holds [11, Theorem 3.1.5]:

$$
\left\|v-\mathscr{J}_{h} v\right\|_{m, \Omega^{-}} \leq c h^{l-m}\|v\|_{l, \Omega^{-}}, \quad m=0 \text { or } 1 .
$$

Next, we state the following result which can be derived in the same way as in [22, Theorem 3.2].

Lemma 4.1. Let $\rho$ be a real number $\geq 1$ such that $\rho-1 / 2$ is not an integer. There exists a projection operator $\Pi_{N}^{\rho}$ from the space $\left\{v \in H^{\rho}\left(\Omega^{+}\right) ; v=0\right.$ on $\left.\partial \Omega^{+} \backslash \gamma\right\}$ onto $\left\{v_{N} \in Q_{N}\left(\bar{\Omega}^{+}\right) ; v_{N}=0\right.$ on $\left.\partial \Omega^{+} \backslash \gamma\right\}$ such that, if a function $v$ vanishing on $\partial \Omega^{+} \backslash \gamma$ belongs to $H^{\sigma}\left(\Omega^{+}\right)$for a real number $\sigma \geq \rho$, the following error estimate holds:

$$
\left\|v-\Pi_{N}^{\rho} v\right\|_{\mu, \Omega^{+}} \leq c N^{\mu-\sigma}\|v\|_{\sigma, \Omega^{+}}, \quad 0 \leq \mu \leq \rho .
$$

We are now going to approximate a function $u$ of $H_{0}^{1}(\Omega)$, which is sufficiently smooth, by a function $v_{\delta}$, with $v_{\delta}^{*}$ in $V_{\delta}$. We set

$$
v_{\delta}^{*}=\left(\mathscr{F}_{h} u^{-}+q_{h}, \Pi_{N}^{\rho} u^{+}\right) \text {, }
$$

where $q_{h}$ will be chosen in $X_{h}$ so that $v_{\delta}$ satisfies the matching condition under consideration.

4.1. Pointwise matching. We immediately prove the following result.

Proposition 4.2. For any function $u$ in $H_{0}^{1}(\Omega)$ such that the pair $u^{*}$ belongs to $H^{l}\left(\Omega^{-}\right) \times H^{\sigma}\left(\Omega^{+}\right)$, where $l$ and $\sigma$ are real numbers, $2 \leq l \leq k+1$ and $\sigma \geq 2$, there exists a pair $v_{\delta}^{*}$ in $V_{\delta}^{P}$ such that

$$
\left\|u^{*}-v_{\delta}^{*}\right\| \leq c\left\{h^{l-1}\left\|u^{-}\right\|_{l, \Omega^{-}}+\left(h^{\sigma-1}+N^{1-\sigma}\right)\left\|u^{+}\right\|_{\sigma, \Omega^{+}}\right\} .
$$


Proof. For any function $z$ defined on $\Omega^{+}$, denote by $\bar{z}$ the function defined on $\Omega^{-}$by

$$
\bar{z}(x, y)=z(-x, y) \quad \forall(x, y) \in \Omega^{-} .
$$

Next, take $2 \leq \rho \leq \inf \{k+1, \sigma\}, \rho-1 / 2 \notin \mathbb{N}$, and choose $v_{\delta}^{*}$ as in (4.4) with $q_{h}$ equal to $-\mathscr{J}_{h}\left(\bar{u}^{+}-\overline{\Pi_{N}^{\rho} u^{+}}\right)$. Clearly, we have at any point a of $\xi_{h}$,

$$
\left(\mathscr{F}_{h} u^{-}\right)(\mathbf{a})+q_{h}(\mathbf{a})=u^{-}(\mathbf{a})-\bar{u}^{+}(\mathbf{a})+\left({\overline{\Pi_{N}^{\rho}}}^{+}\right)(\mathbf{a})=\left(\Pi_{N}^{\rho} u^{+}\right)(\mathbf{a}),
$$

so that $v_{\delta}^{*}$ belongs to $V_{\delta}^{P}$. Moreover, we write

$$
\begin{aligned}
\left\|u^{*}-v_{\delta}^{*}\right\| \leq & \left\|u^{-}-\mathscr{I}_{h} u^{-}\right\|_{1, \Omega^{-}}+\left\|q_{h}\right\|_{1, \Omega^{-}}+\left\|u^{+}-\Pi_{N}^{\rho} u^{+}\right\|_{1, \Omega^{+}} \\
\leq & \left\|u^{-}-\mathscr{I}_{h} u^{-}\right\|_{1, \Omega^{-}}+\left\|\left(\operatorname{id}-\mathscr{F}_{h}\right)\left(\bar{u}^{+}-\bar{\Pi}_{N}^{\rho} u^{+}\right)\right\|_{1, \Omega^{-}} \\
& +2\left\|u^{+}-\Pi_{N}^{\rho} u^{+}\right\|_{1, \Omega^{+}} .
\end{aligned}
$$

Finally, applying (4.2) and Lemma 4.1, we deduce

$$
\begin{aligned}
\left\|u^{*}-v_{\delta}^{*}\right\| & \leq c\left\{h^{l-1}\left\|u^{-}\right\|_{l, \Omega^{-}}+h^{\rho-1}\left\|\bar{u}^{+}-\overline{\Pi_{N}^{\rho} u^{+}}\right\|_{\rho, \Omega^{-}}+N^{1-\sigma}\left\|u^{+}\right\|_{\sigma, \Omega^{+}}\right\} \\
& \leq c\left\{h^{l-1}\left\|u^{-}\right\|_{l, \Omega^{-}}+h^{\rho-1} N^{\rho-\sigma}\left\|u^{+}\right\|_{\sigma, \Omega^{+}}+N^{1-\sigma}\left\|u^{+}\right\|_{\sigma, \Omega^{+}}\right\} .
\end{aligned}
$$

Applying the convexity inequality $\alpha \beta \leq \alpha^{p} / p+\beta^{q} / q(1 / p+1 / q=1, \alpha \geq 0$, $\beta \geq 0)$ gives the proposition.

4.2. Integral matching. As far as the approximation error is concerned, this case is less simple. We are led to make an additional assumption on the family of triangulations: more precisely, we suppose that there exists a positive constant $\sigma$ such that, for any $h$ and for any triangles $K$ and $K^{\prime}$ in $\mathscr{T}_{h}$ such that the lengths of $K \cap \bar{\gamma}$ and of $K^{\prime} \cap \bar{\gamma}$ are positive, the following inequality holds

$$
h_{K} \leq \sigma h_{K^{\prime}} .
$$

That means that the family of triangulations is uniformly regular over $\gamma$. The following result is proven in [7, Lemma 5.1].

Lemma 4.3. If assumption (4.6) is satisfied, there exists a lifting operator $\boldsymbol{R}_{h}$ from $x_{h}$ into $X_{h}$ such that the following estimate holds for any $v_{h}$ in $x_{h}$ :

$$
\left\|R_{h} v_{h}\right\|_{1, \Omega^{-}} \leq c\left\|v_{h}\right\|_{1 / 2^{*}, \gamma} .
$$

We recall that $\pi_{h}$ stands for the orthogonal projection operator from $L^{2}(\gamma)$ onto $x_{h}$. Our goal is to prove a stability result for this operator in the $H_{00}^{1 / 2}$ norm.

Lemma 4.4. If assumption (4.6) is satisfied, the following stability property holds for any function $v$ in $H_{00}^{1 / 2}(\gamma)$ :

$$
\left\|\pi_{h} v\right\|_{1 / 2^{*}, \gamma} \leq c\|v\|_{1 / 2^{*}, \gamma} .
$$

Proof. First, let $v$ be any function in $H_{0}^{1}(\gamma)$. To bound $\left\|\pi_{h} v\right\|_{1, \gamma}$, we write

$$
\left\|\pi_{h} v\right\|_{1, \gamma} \leq\left\|i_{h} v\right\|_{1, \gamma}+\left\|\pi_{h} v-i_{h} v\right\|_{1, \gamma} .
$$


Since the family of the $\left\{K \cap \bar{\gamma}, K \in \mathscr{T}_{h}\right\}$ is uniformly regular, denoting by $\tilde{h}$ the maximum of the lengths of $K \cap \bar{\gamma}, K \in \mathscr{T}_{h}$, we can apply the standard inverse inequality [11, Theorem 3.2.6], which gives

$$
\begin{aligned}
\left\|\pi_{h} v\right\|_{1, \gamma} & \leq\left\|i_{h} v\right\|_{1, \gamma}+c \tilde{h}^{-1}\left\|\pi_{h} v-i_{h} v\right\|_{0, \gamma} \\
& \leq\|v\|_{1, \gamma}+\left\|v-i_{h} v\right\|_{1, \gamma}+c \tilde{h}^{-1}\left(\left\|v-i_{h} v\right\|_{0, \gamma}+\left\|v-\pi_{h} v\right\|_{0, \gamma}\right) .
\end{aligned}
$$

Using the estimate for the interpolation error [11, Theorem 3.15] together with (3.5), we obtain

$$
\left\|\pi_{h} v\right\|_{1, \gamma} \leq c\|v\|_{1, \gamma} .
$$

Finally, by the definition of the space $H_{00}^{1 / 2}(\gamma)$, we interpolate this result with the estimate

$$
\left\|\pi_{h} v\right\|_{0, \gamma} \leq\|v\|_{0, \gamma}
$$

with index $1 / 2$ (cf. [20, Chapter 1, Theorem 5.1]) and we obtain the desired result.

Remark 4.5. It is known that the stability result (4.8) holds if a less restrictive assumption than (4.6) is satisfied. However, the necessary condition for (4.7) to be true is not clear.

Proposition 4.6. If assumption (4.6) is satisfied, for any function $u$ in $H_{0}^{1}(\Omega)$ such that the pair $u^{*}$ belongs to $H^{l}\left(\Omega^{-}\right) \times H^{\sigma}\left(\Omega^{+}\right)$, where $l$ and $\sigma$ are real numbers, $2 \leq l \leq k+1$ and $\sigma \geq 2$, there exists a pair $v_{\delta}^{*}$ in $V_{\delta}^{I}$ such that

$$
\left\|u^{*}-v_{\delta}^{*}\right\| \leq c\left\{h^{l-1}\left\|u^{-}\right\|_{l, \Omega^{-}}+N^{1-\sigma}\left\|u^{+}\right\|_{\sigma, \Omega^{+}}\right\} \text {. }
$$

Proof. We choose $v_{\delta}$ as in (4.4) with $\rho$ equal to 1 and $q_{h}$ equal to

$$
R_{h} \pi_{h}\left(\Pi_{N}^{1} u^{+}-\mathscr{I}_{h} u^{-}\right) \text {. }
$$

Then, we have

$$
\mathscr{I}_{h} u^{-}+q_{h}-\pi_{h} \Pi_{N}^{1} u^{+}=0,
$$

so that $v_{\delta}^{*}$ belongs to $V_{\delta}^{I}$. Moreover, we estimate

$$
\begin{aligned}
\left\|u^{*}-v_{\delta}^{*}\right\| \leq & \left\|u^{-}-\mathscr{I}_{h} u^{-}\right\|_{1, \Omega^{-}}+\left\|R_{h} \pi_{h}\left(\Pi_{N}^{1} u^{+}-\mathscr{I}_{h} u^{-}\right)\right\|_{1, \Omega^{-}} \\
& +\left\|u^{+}-\Pi_{N}^{1} u^{+}\right\|_{1, \Omega^{+}} \\
\leq & \left\|u^{-}-\mathscr{I}_{h} u^{-}\right\|_{1, \Omega^{-}}+\left\|R_{h} \pi_{h}\left(u^{-}-\mathscr{I}_{h} u^{-}\right)\right\|_{1, \Omega^{-}} \\
& +\left\|R_{h} \pi_{h}\left(u^{+}-\Pi_{N}^{1} u^{+}\right)\right\|_{1, \Omega^{-}}+\left\|u^{+}-\Pi_{N}^{1} u^{+}\right\|_{1, \Omega^{+}} .
\end{aligned}
$$

Lemmas 4.3 and 4.4 imply

$$
\begin{aligned}
\left\|u^{*}-v_{\delta}^{*}\right\| \leq & \left\|u^{-}-\mathscr{I}_{h} u^{-}\right\|_{1, \Omega^{-}}+c\left(\left\|u^{-}-\mathscr{I}_{h} u^{-}\right\|_{1 / 2^{*}, \gamma}+\left\|u^{+}-\Pi_{N}^{1} u^{+}\right\|_{1 / 2^{*}, \gamma}\right) \\
& +\left\|u^{+}-\Pi_{N}^{1} u^{+}\right\|_{1, \Omega^{+}} .
\end{aligned}
$$


Using the trace theorem, we obtain simply

$$
\left\|u^{*}-v_{\delta}^{*}\right\| \leq c\left(\left\|u^{-}-\mathscr{I}_{h} u^{-}\right\|_{1, \Omega^{-}}+\left\|u^{+}-\Pi_{N}^{1} u^{+}\right\|_{1, \Omega^{+}}\right) .
$$

Finally, using the estimates (4.2) and (4.3) gives the result.

Remark 4.7. Here also, the error is better for the integral matching condition than for the pointwise one. Indeed, in (4.10), the two discretization parameters enter in a completely independent way.

\section{FinAl estimates AND CONCLUSION}

First, we recall an estimate which follows at once from a standard result in spectral methods [10, Lemma $3.2 ; 24,(3.22)]$, in a slightly improved form due to [6, Appendix B].

Lemma 5.1. For any function $f$ in $L^{2}(\Omega)$ such that the function $\left(y-y^{2}\right) f^{+}$ belongs to $H^{\rho}\left(\Omega^{+}\right)$, where $\rho$ is a real number $>1$, the following estimate holds for any $w_{\delta}^{*}=\left(w_{h}, w_{N}\right)$ in $V_{\delta}$ :

$$
\left(f^{*}, w_{\delta}^{*}\right)-\left(f^{*}, w_{\delta}^{*}\right)_{\delta} \leq c N^{1-\rho}\left\|\left(y-y^{2}\right) f^{+}\right\|_{\rho, \Omega^{+}}\left\|w_{N}\right\|_{1, \Omega^{+}} .
$$

Our main results are stated in the two following theorems.

Theorem 5.2. Assume that the solution $u$ of problem (1.1) is such that the pair $u^{*}$ belongs to $H^{2}\left(\Omega^{-}\right) \times H^{\sigma}\left(\Omega^{+}\right)$, where $\sigma$ is a real number $\geq 2$. Assume moreover that the function $f$ of $L^{2}(\Omega)$ is such that the function $\left(y-y^{2}\right) f^{+}$ belongs to $H^{\rho}\left(\Omega^{+}\right)$, where $\rho$ is a real number $>1$. Then, in the case of the pointwise matching condition, the solutions $u$ and $u_{\delta}$ of problems (1.1) and (2.15) satisfy

$$
\begin{aligned}
\left\|u^{*}-u_{\delta}^{*}\right\| \leq c\left\{h^{1 / 2} \sqrt{\left|\log \left(h N^{2}\right)\right| \| u^{-}} \|_{2, \Omega^{-}}\right. & +\left(h^{\sigma-1}+N^{1-\sigma}\right)\left\|u^{+}\right\|_{\sigma, \Omega^{+}} \\
& \left.+N^{1-\rho}\left\|\left(y-y^{2}\right) f^{+}\right\|_{\rho, \Omega^{+}}\right\} .
\end{aligned}
$$

Theorem 5.3. Assume that the solution $u$ of problem (1.1) is such that the pair $u^{*}$ belongs to $H^{l}\left(\Omega^{-}\right) \times H^{\sigma}\left(\Omega^{+}\right)$, where $l$ and $\sigma$ are real numbers, $2 \leq l \leq k+1$ and $\sigma \geq 2$. Assume moreover that assumption (4.6) is satisfied and that the function $f$ of $L^{2}(\Omega)$ is such that the function $\left(y-y^{2}\right) f^{+}$belongs to $H^{\rho}\left(\Omega^{+}\right)$, where $\rho$ is a real number $>1$. Then, in the case of the integral matching condition, the solutions $u$ and $u_{\delta}$ of problems (1.1) and (2.15) satisfy

$$
\left\|u^{*}-u_{\delta}^{*}\right\| \leq c\left\{h^{l-1}\left\|u^{-}\right\|_{l, \Omega^{-}}+N^{1-\sigma}\left\|u^{+}\right\|_{\sigma, \Omega^{+}}+N^{1-\rho}\left\|\left(y-y^{2}\right) f^{+}\right\|_{\rho, \Omega^{+}}\right\} .
$$

Proof. We set $\tilde{\delta}=\left(h,(N-1)^{-1}\right)$. Of course, we apply Proposition 2.5 and, in (2.20), we choose $v_{\delta}^{*}=\left(v_{h}, v_{N}\right)$ equal to the pair defined in Propositions 4.2 and 4.6 , respectively, but with $\delta$ replaced by $\tilde{\delta}$. Since $v_{N}$ belongs to $Q_{N-1}\left(\Omega^{+}\right)$and the quadrature formula $(2.12)$ is exact for all polynomials of degree $\leq 2 N-1$, this implies that, for any $w_{\delta}^{*}$ in $V_{\delta}$,

$$
a\left(v_{\delta}^{*}, w_{\delta}^{*}\right)=a_{\delta}\left(v_{\delta}^{*}, w_{\delta}^{*}\right) .
$$


Then the estimates (5.2) and (5.3) follow from (2.20), Propositions 3.1 and 3.4, respectively, Propositions 4.2 and 4.6, respectively, and Lemma 5.1.

By a classical duality method, it is possible to derive an improved estimate for $\left\|u-u_{\delta}\right\|_{0, \Omega}$ in the case of the integral matching condition.

Proposition 5.4. Under the assumptions of Theorem 5.3, in the case of the integral matching condition, the solutions $u$ and $u_{\delta}$ of problems (1.1) and (2.15) satisfy

$$
\begin{aligned}
\left\|u-u_{\delta}\right\|_{0, \Omega} \leq c\left\{h^{l-1}\left(h+N^{-1}\right)\left\|u^{-}\right\|_{l, \Omega^{-}}\right. & N^{1-\sigma}\left(h+N^{-1}\right)\left\|u^{+}\right\|_{\sigma, \Omega^{+}} \\
& \left.+N^{1-\rho}\left\|\left(y-y^{2}\right) f^{+}\right\|_{\rho, \Omega^{+}}\right\} .
\end{aligned}
$$

Proof. We have

$$
\left\|u-u_{\delta}\right\|_{0, \Omega}=\sup _{g \in L^{2}(\Omega)} \int_{\Omega}\left(u-u_{\delta}\right)(\mathbf{x}) g(\mathbf{x}) d \mathbf{x} /\|g\|_{0, \Omega} .
$$

Let $g$ be any function in $L^{2}(\Omega)$. The unique solution $w$ in $H_{0}^{1}(\Omega)$ of the problem

$$
-\Delta w=g \quad \text { in } \Omega, \quad w=0 \text { on } \partial \Omega
$$

satisfies

$$
\|w\|_{2, \Omega} \leq c\|g\|_{0, \Omega}
$$

Setting $u_{\delta}^{*}=\left(u_{h}, u_{N}\right)$, we compute

$$
\int_{\Omega}\left(u-u_{\delta}\right)(\mathbf{x}) g(\mathbf{x}) d \mathbf{x}=a\left(u^{*}-u_{\delta}^{*}, w^{*}\right)+\int_{\gamma}(\partial w / \partial n)(0, y)\left(u_{N}-u_{h}\right)(0, y) d y .
$$

Hence, for any $w_{\delta}^{*}$ in $V_{\tilde{\delta}}$, with $\tilde{\delta}=\left(h,(N-1)^{-1}\right)$, we have

$$
\begin{aligned}
\int_{\Omega}\left(u-u_{\delta}\right)(\mathbf{x}) g(\mathbf{x}) d \mathbf{x}= & a\left(u^{*}-u_{\delta}^{*}, w^{*}-w_{\delta}^{*}\right)+\left(f^{*}, w_{\delta}^{*}\right)-\left(f^{*}, w_{\delta}^{*}\right)_{\delta} \\
& +\int_{\gamma}(\partial w / \partial n)(0, y)\left(u_{N}-u_{h}\right)(0, y) d y
\end{aligned}
$$

Choosing $w_{\delta}^{*}$ as defined in Proposition 4.6, and using Lemma 5.1, we obtain

$$
\begin{aligned}
\int_{\Omega}(u & \left.-u_{\delta}\right)(\mathbf{x}) g(\mathbf{x}) d \mathbf{x} \\
\leq & c\left\{\left(h+N^{-1}\right)\left\|u^{*}-u_{\delta}^{*}\right\|+N^{1-\rho}\left\|\left(y-y^{2}\right) f^{*}\right\|_{\rho, \Omega^{+}}\right\}\|w\|_{2, \Omega} \\
& +\int_{\gamma}(\partial w / \partial n)(0, y)\left(u_{N}-u_{h}\right)(0, y) d y .
\end{aligned}
$$


It remains to estimate the last term in (5.7). We note that $u_{h \mid \gamma}$ is equal to $\pi_{h} u_{N}$, so that

$$
\begin{aligned}
\int_{\gamma}(\partial w / \partial n)(0, y)\left(u_{N}-u_{h}\right)(0, y) d y \\
\quad=\int_{\gamma}\left[(\partial w / \partial n)-\pi_{h}(\partial w / \partial n)\right](0, y)\left(u_{N}-\pi_{h} u_{N}\right)(0, y) d y \\
\quad=\int_{\gamma}\left[(\partial w / \partial n)-\pi_{h}(\partial w / \partial n)\right](0, y)\left[\left(u-\pi_{h} u\right)-\left(\mathrm{id}-\pi_{h}\right)\left(u-u_{N}\right)\right](0, y) d y \\
\quad \leq\left\|(\partial w / \partial n)-\pi_{h}(\partial w / \partial n)\right\|_{0, \gamma}\left(\left\|u-\pi_{h} u\right\|_{0, \gamma}+\left\|\left(\mathrm{id}-\pi_{h}\right)\left(u-u_{N}\right)\right\|_{0, \gamma}\right) .
\end{aligned}
$$

Using (3.5) and (3.6) yields

$$
\begin{aligned}
& \int_{\gamma}(\partial w / \partial n)(0, y)\left(u_{N}-u_{h}\right)(0, y) d y \\
& \quad \leq c h^{1 / 2}\|\partial w / \partial n\|_{1 / 2^{*}, \gamma}\left(h^{l-1 / 2}\left\|u^{-}\right\|_{l, \Omega^{-}}+h^{1 / 2}\left\|u^{+}-u_{N}\right\|_{1, \Omega^{+}}\right),
\end{aligned}
$$

which, together with (5.6) and (5.7), gives (5.4).

The detailed analysis we have performed allows us to compare the two algorithms, corresponding to different matching conditions. Indeed, whatever the regularity of the exact solution is, we obtain better convergence results in the case of the integral matching condition. Since we have already noted that the computational cost of the two methods is of the same order, we believe that this last algorithm has to be preferred. Numerical tests [14,19] which are currently being implemented are expected to confirm the theoretical results.

As already stated, in this paper we are only concerned with a model problem on a model domain. However, in this very simple example, it turns out that the order of accuracy in the finite element domain is simultaneously restricted by the degree of polynomials and by the regularity of the solution, while in the spectral domain it is only limited by the regularity of the solution. That is why we believe that, in more general elliptic problems (for instance the Stokes or NavierStokes equations), the finite element domain must be chosen in such a way that it contains a neighborhood of both the singularities of the solution and the singularities of the boundary of the domain (for instance, corners of polygons, which induce singularities of the solution even if the right-hand member is very smooth). Then, local refinements of the mesh can be applied to improve the convergence, in a much simpler way than for the $p$-version of finite elements. These techniques are presently being developed in [14]. It is important to note here that the balance between the finite element subdomain and the spectral element subdomain is much more easy to handle than a local refinement in the so-called $h-p$ version of finite elements. The matching between the two domains is indeed less stringent in the present method than in the $h-p$ version, where the intersection of two different domains is either a whole edge, or a vertex, or empty.

We conclude this paper by giving an example of a domain with a less trivial boundary, and by explaining how to choose the parameters in this case. 
Example. Let $\Omega$ be a polygonal domain with vertices $\mathbf{a}_{i}, 1 \leq i \leq K$. For $1 \leq i \leq K$, we denote by $\omega_{i}$ the measure of the interior angle at $\mathbf{a}_{i}$ and by $d_{i}(\Delta)$ the distance of any closed set $\Delta$ in $\bar{\Omega}$ to $\mathbf{a}_{i}$. The domain $\Omega^{+}$is chosen as a large rectangle contained in $\Omega$, such that $\bar{\Omega}$ does not contain any vertex $\mathbf{a}_{i}, 1 \leq i \leq K$; then $\Omega^{-}$is defined as $\Omega \backslash \bar{\Omega}^{+}$(cf. Figure 5.1).

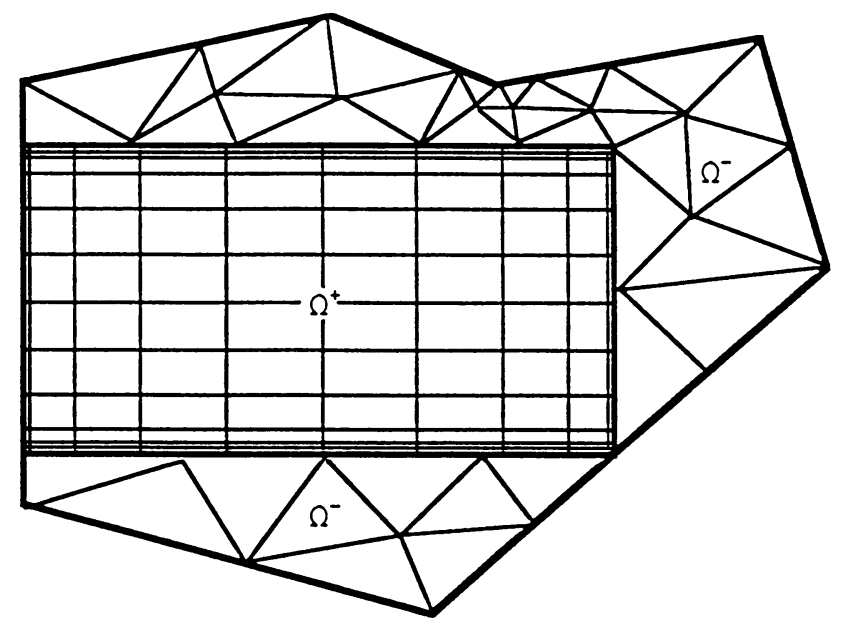

FIGURE 5.1. An example of a domain with a less trivial boundary.

In the domain $\Omega$, we solve the problem (1.1). It is known (see [17, Theorem 5.1.3.5] or [27, Theorem 1]) that, if $f$ belongs to $H^{m}(\Omega)$ for a real number $m \geq 0$, the solution $u$ satisfies:

(1) The function $u^{-}$belongs to the space

$W_{\rho}^{2}\left(\Omega^{-}\right)=\left\{v: \Omega^{-} \rightarrow \mathbb{R} ; \int_{\Omega^{-}}\left(\partial^{j+k} v / \partial x^{j} \partial y^{k}\right)^{2} \rho(\mathbf{x}) d \mathbf{x}<+\infty, 0 \leq j+k \leq 2\right\}$,

where $\rho(\mathbf{x})$ is a positive bounded weight equivalent to $d_{i}(\mathbf{x})^{2 \alpha_{i}}$ in a neighborhood of $\mathbf{a}_{i}, 1 \leq i \leq K$, with

moreover, one has

$$
\left\{\begin{array}{l}
\alpha_{i}=0 \quad \text { if } \omega_{i}<\pi \\
\alpha_{i}>1-\pi / \omega_{i} \text { if } \omega_{i}>\pi
\end{array}\right.
$$

$$
\left(\sum_{0 \leq j+k \leq 2} \int_{\Omega^{-}}\left(\partial^{j+k} v / \partial x^{j} \partial y^{k}\right)^{2} \rho(\mathbf{x}) d \mathbf{x}\right)^{1 / 2} \leq c\|f\|_{0, \Omega} .
$$

(2) The function $u^{+}$belongs to the space $H^{m+2}\left(\Omega^{+}\right)$, and one has

$$
\|u\|_{m+2, \Omega^{+}} \leq c\left(\inf _{1 \leq i \leq K} d_{i}\left(\bar{\Omega}^{+}\right)^{\pi / \omega_{i}-m-1}\right)\|f\|_{m, \Omega} .
$$

Next, we consider a regular family of triangulations $\left(\mathscr{T}_{h}\right)_{h}$ of $\Omega^{-}$such that, for any $\omega_{i}>\pi, 1 \leq i \leq K$, the diameter of each triangle containing the vertex 
$\mathbf{a}_{i}$ is bounded by $c h^{1 /\left(1-\alpha_{i}\right)}$ and the diameter of each triangle $K$ contained in a fixed neighborhood of $\mathbf{a}_{i}$ is bounded by $c h d_{i}(K)$; a way of constructing such a triangulation was first described in [27]. The finite element functions are chosen to be piecewise linear continuous functions (i.e., $k$ is equal to 1). Using [27, Theorem 2] together with Theorem 5.3, we see that the discrete solution $u_{\delta}$ of problem (2.15) satisfies the error estimate

$$
\left\|u^{*}-u_{\delta}^{*}\right\| \leq c(f)\left\{h+N^{-m-1}\left(\inf _{1 \leq i \leq K} d_{i}^{\pi / \omega_{i}-m-1}\right)+N^{-m}\right\} .
$$

Of course, in a practical computation, the parameters will be chosen so as to make the three error terms $h, N^{-m-1}\left(\inf _{1 \leq i \leq K} d_{i}^{\pi / \omega_{i}-m-1}\right)$, and $N^{-m}$ of the same order. For instance, assume that $m$ is equal to 3 , that the $d_{i}$ are equal to $2 \cdot 10^{-1}$, and that the constants are bounded by 10 ; in order to obtain a precision of $10^{-2}$, we choose $h$ equal to $10^{-3}$ and $N$ equal to $23 \simeq\left(10^{3 / 4}\right) \cdot\left(5^{7 / 8}\right)$, which requires $\mathscr{O}\left(10^{6}\right)$ operations in the finite element domain and $\mathscr{O}\left(1.2 \times 10^{4}\right)$ in the spectral domain; in order to obtain a precision of $10^{-3}$, we choose $h$ equal to $10^{-4}$ and $N$ equal to $41 \simeq 10 \cdot\left(5^{7 / 8}\right)$, which requires $\mathscr{O}\left(10^{8}\right)$ operations in the finite element domain and $\mathscr{O}\left(7 \times 10^{4}\right)$ in the spectral domain.

\section{ACKNOWLEDGMENTS}

We are very grateful to Professor J.-L. Lions who agreed to present a note about our work to the Académie des Sciences of Paris. We are also greatly indebted to Vivette Girault for her kind and helpful advice.

\section{BIBLIOGRAPHY}

1. I. Babuška, B. Szabo, and I. N. Katz, The p-version of the finite element method, SIAM J. Numer. Anal. 18 (1981), 515-545.

2. J. Berg and J. Löfström, Interpolation spaces: An introduction, Springer-Verlag, Berlin, Heidelberg, and New York, 1976.

3. C. Bernardi, Optimal finite-element interpolation on curved domains, SIAM J. Numer. Anal. 26 (1989), 1212-1240.

4. __ Interpolation par éléments finis de fonctions tout juste continues et application (in preparation).

5. C. Bernardi, N. Debit, and Y. Maday, Couplage de méthodes spectrale et d'éléments finis: premiers résultats d'approximation, C. R. Acad. Sci. Paris Sér. I Math. 305 (1987), 353-356.

6. C. Bernardi and Y. Maday, Some spectral approximations of one-dimensional fourth-order problems, J. Approx. Theory (to appear).

7. P. E. Bjørstad and O. B. Widlund, Iterative methods for the solution of elliptic problems on regions partitioned into substructures, SIAM J. Numer. Anal. 23 (1986), 1097-1020.

8. C. Canuto, M. Y. Hussaini, A. Quarteroni, and T. A. Zang, Spectral methods with applications to fluid dynamics, Springer-Verlag, Berlin, Heidelberg, and New York, 1987.

9. C. Canuto and A. Quarteroni, Approximation results for orthogonal polynomials in Sobolev spaces, Math. Comp. 38 (1982), 67-86.

10. __ Spectral and pseudo-spectral methods for parabolic problems with nonperiodic boundary conditions, Calcolo 18 (1981), 197-218.

11. P. G. Ciarlet, The finite element method for elliptic problems, North-Holland, Amsterdam, New York, and Oxford, 1978. 
12. M. Crouzeix and P.-A. Raviart, Conforming and nonconforming finite element methods for solving the stationary Stokes equations. I, RAIRO 7-R3 (1973), 33-75.

13. P. J. Davis and P. Rabinowitz, Methods of numerical integration, Academic Press, Orlando, 1985.

14. N. Debit, Thesis (in preparation).

15. D. Funaro, A multidomain spectral approximation of elliptic equations, Numer. Methods Partial Differential Equations 2 (1986), 187-205.

16. D. Gottlieb and S. A. Orszag, Numerical analysis of spectral methods, SIAM CBMS, Philadelphia, 1977.

17. P. Grisvard, Elliptic problems in nonsmooth domains, Pitman, Boston, London, and Melbourne, 1985.

18. Handbook of mathematical functions (M. Abramowitz and I. A. Stegun, eds.), Dover, New York, 1970.

19. K. Z. Korczak and A. T. Patera, An isoparametric spectral element method and its application to incompressible two-dimensional flow in complex geometries, Proc. 6th GAMM Conference on Numerical Methods in Fluid Mechanics, Vieweg, 1986.

20. J.-L. Lions and E. Magenes, Problèmes aux limites non homogènes et applications, Vols. I \& II, Dunod, Paris, 1968.

21. Y. Maday, Analysis of spectral projectors in one-dimensional domains, Math. Comp. (to appear).

22. __ Analysis of spectral projectors in multi-dimensional domains, Math. Comp. (to appear).

23. Y. Maday and A. T. Patera, Spectral element methods for the incompressible Navier-Stokes equations, State of the Art Surveys in Computational Mechanics (A. Noor and J. T. Oden, eds.), A.S.M.E., 1989, pp. 71-143.

24. Y. Maday and A. Quarteroni, Legendre and Chebyshev spectral approximations of Burgers' equation, Numer. Math 37 (1981), 321-332.

25. A. T. Patera, A spectral element method for fluid dynamics: laminar flow in a channel expansion, J. Comput. Phys. 54 (1984), 468-488.

26. A. Quarteroni, Some results of Bernstein and Jackson type for polynomial approximation in $\mathbf{L}^{p}$-spaces, Japan J. Appl. Math. 1 (1984), 173-181.

27. G. Raugel, Résolution numérique par une méthode d'éléments finis du problème de Dirichlet pour le laplacien dans un polygone, C.R. Acad. Sci. Paris Sér. A 286 (1978), 791-794.

28. P. A. Raviart and J. M. Thomas, Primal hybrid finite element methods for 2 nd order elliptic equations, Math. Comp. 31 (1977), 391-413.

29. L. R. Scott and M. Vogelius, Norm estimates for a maximal right inverse of the divergence operator in spaces of piecewise polynomials, Model. Math. Anal. Numér. 19 (1985), 111-143.

30. G. Szegö, Orthogonal polynomials, Colloq. Publ., vol. 23, Amer. Math. Soc., Providence, R.I., 1978.

31. G. Talenti, Best constant in Sobolev inequality, Ann. Mat. Pura Appl. (4) 110 (1976), 253-272.

32. M. Vogelius, A right-inverse for the divergence operator in spaces of piecewise polynomials. Application to the p-version of the finite element method, Numer. Math. 41 (1983), 19-37.

Laboratoire d'Analyse Numérique, Université Pierre et Marie Curie, 4 Place Jussieu, 75252 Paris Cedex 05, France 